\title{
Hydrogen sulfide ameliorates aging-associated changes in the kidney
}

\author{
Hak Joo Lee • Denis Feliers • Jeffrey L. Barnes • Sae Oh • Goutam Ghosh Choudhury • \\ Vivian Diaz • Veronica Galvan • Randy Strong • James Nelson • Adam Salmon • \\ Christopher G. Kevil • Balakuntalam S. Kasinath
}

Received: 23 March 2018 / Accepted: 9 April 2018 / Published online: 1 May 2018

(C) This is a U.S. Government work and not under copyright protection in the US; foreign copyright protection may apply 2018

\begin{abstract}
Aging is associated with replacement of normal kidney parenchyma by fibrosis. Because hydrogen sulfide $\left(\mathrm{H}_{2} \mathrm{~S}\right)$ ameliorates kidney fibrosis in disease models, we examined its status in the aging kidney. In the first study, we examined kidney cortical $\mathrm{H}_{2} \mathrm{~S}$ metabolism and signaling pathways related to synthesis of proteins including matrix proteins in young and old male $\mathrm{C} 57 \mathrm{BL} / 6$ mice. In old mice, increase in renal cortical content of matrix protein involved in fibrosis was associated with decreased $\mathrm{H}_{2} \mathrm{~S}$ generation and AMPK activity, and activation of insulin receptor (IR)/ IRS-2-Akt-mTORC1-mRNA translation signaling axis that can lead to increase in protein synthesis. In the second study, we randomized 18-19 month-old male
\end{abstract}

An abstract containing the data in this manuscript was presented at the annual meeting of the American Society of Nephrology, New Orleans, LA, in November 2017.

Electronic supplementary material The online version of this article (https://doi.org/10.1007/s11357-018-0018-y) contains supplementary material, which is available to authorized users.

H. J. Lee • D. Feliers · J. L. Barnes • S. Oh •

G. G. Choudhury $\cdot$ B. S. Kasinath $(\bowtie)$

Department of Medicine, University of Texas Health San Antonio, 7703, Floyd Curl Drive, MC7882, San Antonio, TX 78229, USA

e-mail: kasinath@uthscsa.edu

J. L. Barnes · G. G. Choudhury · V. Galvan · R. Strong • A. Salmon · B. S. Kasinath

South Texas Veterans Health Care System, San Antonio, TX, USA
C57BL/6 mice to receive $30 \mu \mathrm{mol} / \mathrm{L}$ sodium hydrosulfide (NaHS) in drinking water vs. water alone (control) for 5 months. Administration of NaHS increased plasma free sulfide levels. NaHS inhibited the increase in kidney cortical content of matrix proteins involved in fibrosis and ameliorated glomerulosclerosis. NaHS restored AMPK activity and inhibited activation of IR/IRS-2-Akt-mTORC1-mRNA translation axis. NaHS inhibited age-related increase in kidney cortical content of $\mathrm{p} 21$, IL- $1 \beta$, and IL-6, components of the senescence-associated secretory phenotype. NaHS abolished increase in urinary albumin excretion seen in control mice and reduced serum cystatin $\mathrm{C}$ levels suggesting improved glomerular clearance function. We

V. Diaz · V. Galvan · R. Strong · J. Nelson · A. Salmon •

B. S. Kasinath

Barshop Institute for Longevity and Aging Studies, University of Texas Health, San Antonio, TX, USA

A. Salmon

Department of Molecular Medicine, University of Texas Health San Antonio, San Antonio, TX, USA

C. G. Kevil

Louisiana State University Health Science Center, Shreveport, LA, USA 
conclude that aging-induced changes in the kidney are associated with $\mathrm{H}_{2} \mathrm{~S}$ deficiency. Administration of $\mathrm{H}_{2} \mathrm{~S}$ ameliorates aging-induced kidney changes probably by inhibiting signaling pathways leading to matrix protein synthesis.

Keywords Insulin receptor signaling · Protein chemistry · Mechanistic target of rapamycin · AMPactivated protein kinase

\section{Introduction}

Gases are important signaling molecules that can affect a wide variety of physiological functions. The role of nitric oxide and carbon monoxide in mammalian physiology is better understood than that of hydrogen sulfide $\left(\mathrm{H}_{2} \mathrm{~S}\right.$ ) (Wang 2012). $\mathrm{H}_{2} \mathrm{~S}$ is a physiologically relevant gasotransmitter synthesized by cystathionine $\gamma$-lyase (CSE), cystathionine $\beta$-synthase (CBS), and 3mercaptopyruvate sulfurtransferase (Feliers et al. 2016; Paul and Snyder 2012). $\mathrm{H}_{2} \mathrm{~S}$ regulates neurotransmission, cell survival, blood pressure, angiogenesis, and response to oxidative stress (Abe and Kimura 1996; Feliers et al. 2016; Paul and Snyder 2012; Yang et al. 2008). Recent work from our group has shown that $\mathrm{H}_{2} \mathrm{~S}$ also affects protein metabolism in the kidney, which has implications for kidney fibrosis, the final common pathway leading to kidney dysfunction in the setting of aging or kidney diseases. In kidney epithelial cells, $\mathrm{H}_{2}$ $\mathrm{S}$ inhibits high glucose-induced protein synthesis that contributes to hypertrophy and increased matrix protein expression; the latter contributes to kidney fibrosis (Lee et al. 2012). Sodium hydrosulfide (NaHS), a source of $\mathrm{H}_{2} \mathrm{~S}$, ameliorates the accumulation of matrix proteins that contributes to kidney fibrosis in diabetic rodents (Sen and Pushpakumar 2016; Yuan et al. 2011; Zhou et al. 2014). We have previously reported that increase in matrix proteins such as collagen leading to fibrosis is also a characteristic feature of the aging kidney (Sataranatarajan et al. 2012); however, the role played by $\mathrm{H}_{2} \mathrm{~S}$ in renal senescence and fibrosis is not well studied. Dietary restriction, a well-established means of extending life span and health span, provides protection against ischemic injury in liver and kidney in an $\mathrm{H}_{2}$ S-dependent manner (Hine et al. 2015). These findings led to our hypothesis that $\mathrm{H}_{2} \mathrm{~S}$ deficiency occurs in the aging kidney and contributes to aging-related changes in its structure and function. We found that $\mathrm{H}_{2} \mathrm{~S}$ deficiency occurs in the aging kidney and that $\mathrm{H}_{2} \mathrm{~S}$ supplementation ameliorates aging-related structural and functional changes in the kidney.

\section{Methods}

Materials

In immunoblotting analyses, we employed antibodies against the following: CBS, CSE, laminin $\gamma 1$, phosphoglycogen synthase kinase $3 \beta($ GSK $\beta)$, GSK $3 \alpha / \beta$ (Santa Cruz Biotechnology Inc, Dallas, TX), phosphop70S6 kinase, p70S6 kinase, phospho-eukaryotic elongation factor F2 (eEF2), eEF2, phospho-eEF2 kinase, eEF2 kinase, phospho-Thr-308-Akt, Akt (Cell Signaling Technology Inc, Danvers, MA), fibronectin, p21, IL-1 $\beta$, IL-6 (Abcam Plc, Cambridge, MA), type I collagen $\alpha 2$ chain (Proteintech Group Inc, Rosemont, IL), and actin (Millipore Sigma, St. Louis, MO). ELISA kits were used to measure changes in phosphorylation of insulin receptor (IR), insulin receptor substrate-2 (IRS2), and acetyl Co-A carboxylase (ACC) (Cell Signaling Technology Inc, Danvers, MA). Analytical kits were used to measure albumin (Bethyl Laboratories Inc, Montgomery, TX), creatinine (Enzo Life Sciences Inc, Farmingdale, NY), and serum cystatin C (R\&D Systems Inc, Minneapolis, MN). Blood glucose concentration was measured by a glucometer (Ascensia Diabetes Care US Inc, Parsippany, NJ).

\section{Animals}

First study We employed cortical lysates from stored kidneys from young male C57BL/6 mice ( $n=10$ mice, mean age 5.2 months) and old male mice ( $n=10$ mice, mean age 30.2 months) to evaluate aging-related changes.

Second study In order to test whether $\mathrm{H}_{2} \mathrm{~S}$ affected aging-related changes in the kidney, we employed male C57BL/6 mice in two batches at the age of 18 and 19 months, respectively (National Institute of Aging). They were housed in a specific pathogen free facility with free access to standard chow and water as previously described (Sataranatarajan et al. 2016). Following initial characterization, equal number of mice was randomized to receive NaHS (Millipore Sigma, St. Louis, $\mathrm{MO})$ in drinking water $(30 \mu \mathrm{mol} / \mathrm{L}$, experimental group $)$ 
or drinking water alone (control group) for 5 months ( $n=10$ in each group in each batch). We chose this dose of $\mathrm{NaHS}$ as it has been shown to increase plasma levels of $\mathrm{H}_{2} \mathrm{~S}$ and ameliorate kidney injury (Sen et al. 2009). $\mathrm{H}_{2} \mathrm{~S}$ content in water was stable for over $24 \mathrm{~h}$ as measured by the colorimetric method described below. Mice in the experimental group received freshly prepared NaHS solution in drinking water every $24 \mathrm{~h}$ whereas those in the control group received fresh water alone. In the first batch, 6 mice from the control group died (4 due to accidental flooding of their cage and 2 from natural causes). Mice in the two batches were sacrificed at the end of 5 months of study at the age of 23 and 24 months, respectively; together, 14 mice in the control group and 20 mice in the experimental group given NaHS were available for analysis. At baseline and at monthly intervals body weight, food and water intake (for 1 week) and blood glucose were measured. Additionally, mice were placed in metabolic cages individually to collect urine for estimation of albumin and creatinine. At sacrifice, a part of the kidney section was fixed in formaldehyde $(4 \% w / v)$ and paraffin embedded for image analysis. The Institutional Animal Care and Utilization Committee of the University of Texas Health at San Antonio approved the animal studies.

Measurement of $\mathrm{H}_{2} \mathrm{~S} \mathrm{H}_{2} \mathrm{~S}$ was measured by the monobromobimane method. Plasma was incubated with excess monobromobimane in $100 \mathrm{mM}$ TrisHCL buffer ( $\mathrm{pH}$ 9.5, 0.1 DTPA) for $30 \mathrm{~min}$ in $1 \%$ oxygen and at room temperature in a hypoxic chamber; the fluorescent product sulfide-dibimane was analyzed by reverse-phase HPLC employing an eclipse XDB-C18 column and $0.1 \%$ trifluoroacetic acid (Millipore Sigma, St. Louis, MO) in acetonitrile as the eluent (Shen et al. 2011). $\mathrm{H}_{2} \mathrm{~S}$ generation was measured by incubating renal cortical lysates with $10 \mathrm{mM}$ L-cysteine (Millipore Sigma, St. Louis, MO) in the presence of $2 \mathrm{mM}$ pyridoxal phosphate (Millipore Sigma, St. Louis, MO) followed by trapping the generated $\mathrm{H}_{2} \mathrm{~S}$ with zinc acetate (Millipore Sigma, St. Louis, MO), as described (Lee et al. 2015).

Morphometric analysis Changes in fractional mesangial area were assessed in periodic acid Schiffstained sections of 27-38 glomeruli from 10 control and 10 NaHS-administered mice as previously described (Sataranatarajan et al. 2012).
Immunoblot analysis Equal amounts of renal cortical protein from homogenates were employed in immunoblot analysis as described (Sataranatarajan et al. 2012). Signal intensities were quantified using the ImageJ software.

Statistical analysis Data were expressed as mean \pm SEM; analyses between two groups were performed by the $t$ test using the GraphPad Prism. Data were considered statistically significant at $p<0.05$.

\section{Results}

Study 1

Kidney cortical content of $\mathrm{H}_{2} \mathrm{~S}$ is reduced in association with increase in matrix protein expression in aging mice In old male mice, the renal cortical expression of $\mathrm{H}_{2} \mathrm{~S}$-synthesizing enzymes CSE and CBS was reduced in association with reduced generation of $\mathrm{H}_{2} \mathrm{~S}$ compared to young mice (Fig. 1a, b). Immunoblotting showed increase in renal cortical expression of matrix proteins, laminin $\gamma 1$, type I collagen $\alpha 2$ chain, and fibronectin (Fig. 1c-e). Laminin $\gamma 1$ is a component of mesangial matrix in the glomerulus; increase in its content contributes to mesangial expansion associated with glomerulosclerosis (Ha et al. 1999). Increase in type I collagen contributes to tubulo-interstitial fibrosis (Sataranatarajan et al. 2012). We and others have previously reported that aging-related increase in matrix proteins is associated with kidney functional impairment leading to increase in urinary albumin excretion and decrease in filtration function (Barsha et al. 2016; Sataranatarajan et al. 2012).

Signaling pathways regulate protein synthesis in the kidney Signaling pathways mediate increase in matrix protein synthesis that contributes to fibrosis of the kidney in disease states (Kasinath et al. 2009). We have previously reported that AMP-activated protein kinase (AMPK) functions as a tonic inhibitor of mTORC1 in the kidney (Kasinath et al. 2009). Inhibition of AMPK removes $\mathrm{mTORC} 1$ inhibition and allows the latter to stimulate mRNA translation and generation of proteins including matrix proteins (Kasinath et al. 2009; Lee et al. 2007). In conditions associated with kidney fibrosis, e.g., diabetes, AMPK is inhibited in association with activation of mTORC1 (Eid et al. 2010; Lee et al. 2007; 
Sataranatarajan et al. 2007; Sharma et al. 2008). In vitro studies have shown that $\mathrm{H}_{2} \mathrm{~S}$ inhibition of high glucoseinduced $\mathrm{mTORC} 1$ activation and matrix protein synthesis is dependent on activating AMPK (Lee et al. 2015; Lee et al. 2012). We explored whether these signaling reactions that promote protein synthesis were activated in the aging kidney.

\section{$A M P K$ is inhibited and mTORC1-regulated mRNA} translation events are stimulated in the aging kidney In old mice kidney, cortical AMPK activity was decreased as shown by reduced Ser-79 phosphorylation of ACC, an AMPK substrate (Fig. 2a). This was associated with increased Thr-389 phosphorylation of p70S6 kinase demonstrating increase in mTORC1 activity (Fig. 2b). p70S6 kinase activation promotes the elongation phase of mRNA translation (Kasinath et al. 2009). Ser-366 phosphorylation of eEF2 kinase by p70S6 kinase inhibits its activity contributing to reduction in Thr-56 phosphorylation of its substrate eEF2 (Redpath et al. 1996). Dephosphorylated eEF2 promotes peptide propagation during elongation phase of translation (Kasinath et al. 2009). Ser-366 phosphorylation of eEF2 kinase was increased and Thr-56 phosphorylation of eEF2 was decreased in the renal cortex of old mice (Fig. 2c, d). These data suggest that in the aging kidney, reduction in AMPK activity is associated with stimulation of mTORC1 and events in protein synthesis that facilitate increase in matrix proteins.

Insulin receptor and Akt are activated in the aging kidney We examined whether Akt is activated in the aging kidney as it is upstream of mTORC1. Renal cortical Akt phosphorylation at Thr-308 was increased in the kidneys of old mice (Fig. 2e). Increase in Ser-9 phosphorylation of GSK $3 \beta$, an Akt phosphorylation site, provided functional confirmation of Akt activation in the aging kidney (Fig. 2f). Because receptor tyrosine kinase activation commonly leads to activation of Akt-mTORC1 signaling and insulin receptor (IR) activation has been linked to aging, we examined the status of IR signaling pathway. Immunoblotting showed increase in tyrosine phosphorylation of IR $\beta$ chain in the renal cortex of old mice, which was further confirmed by an ELISA (Fig. 2g, h). IRS-2 binds activated IR and amplifies its signaling. Tyrosine phosphorylation of IRS-2 was also increased in renal tissues from old mice (Fig. 2i). These data together suggest a potential role for IR in stimulation of AktmTORC1/mTORC2 axis leading to increase in protein synthesis including matrix proteins.

Senescence-associated secretory phenotype occurs in the aging kidney During aging, a subset of cells goes into permanent proliferative arrest termed cell senescence. These senescent cells secrete an array of proteins including pro-inflammatory cytokines that can have injurious autocrine or paracrine effects; this secretome is termed senescence-associated secretory phenotype (SASP). SASP is characterized by increased expression of p21, IL-1 $\beta$, and IL-6 (Acosta et al. 2013). Because mTOR activation has been associated with SASP (Iglesias-Bartolome et al. 2012; Wang et al. 2017), we examined its manifestation in the aging kidney. Kidney cortical expression of p21, IL-1 $\beta$, and IL- 6 was increased in old mice, suggesting that aging kidneys manifested SASP (Fig. 3a-c).

\section{Study 2}

$\mathrm{H}_{2} \mathrm{~S}$ administration is well tolerated by aging mice Having established $\mathrm{H}_{2} \mathrm{~S}$ deficiency occurs in the kidney of old mice, we tested whether its correction by administration of NaHS to 18-19-month-old mice for 5 months ameliorates aging-related kidney dysfunction. At baseline, body weight, food and water consumption, blood glucose concentration, urinary output, and albumin to creatinine ratio were similar between mice randomized to control and NaHS groups (Suppl. Fig. S1AF). During the experimental period of 5 months, serial monthly measurements showed that body weights and consumption of food and water did not differ between the groups (Suppl. Fig. 2A-C). Additionally, blood glucose concentrations were in the normal range in the NaHS and control groups (Suppl. Fig. S2D). At sacrifice, there were no differences in the weights of the kidney, heart, liver, and spleen corrected to either tibial length or body weight (Suppl. Fig. S3A-D). Taken together, these data suggest that NaHS was well tolerated by mice.

$\mathrm{H}_{2} \mathrm{~S}$ inhibits extracellular matrix accumulation in kidneys of aging mice The glomerulus consists of a capillary where glomerular filtration takes place. The mesangium is a structure around which glomerular capillary loops are arranged and it is composed of mesangial cells and extracellular matrix. Aging- 

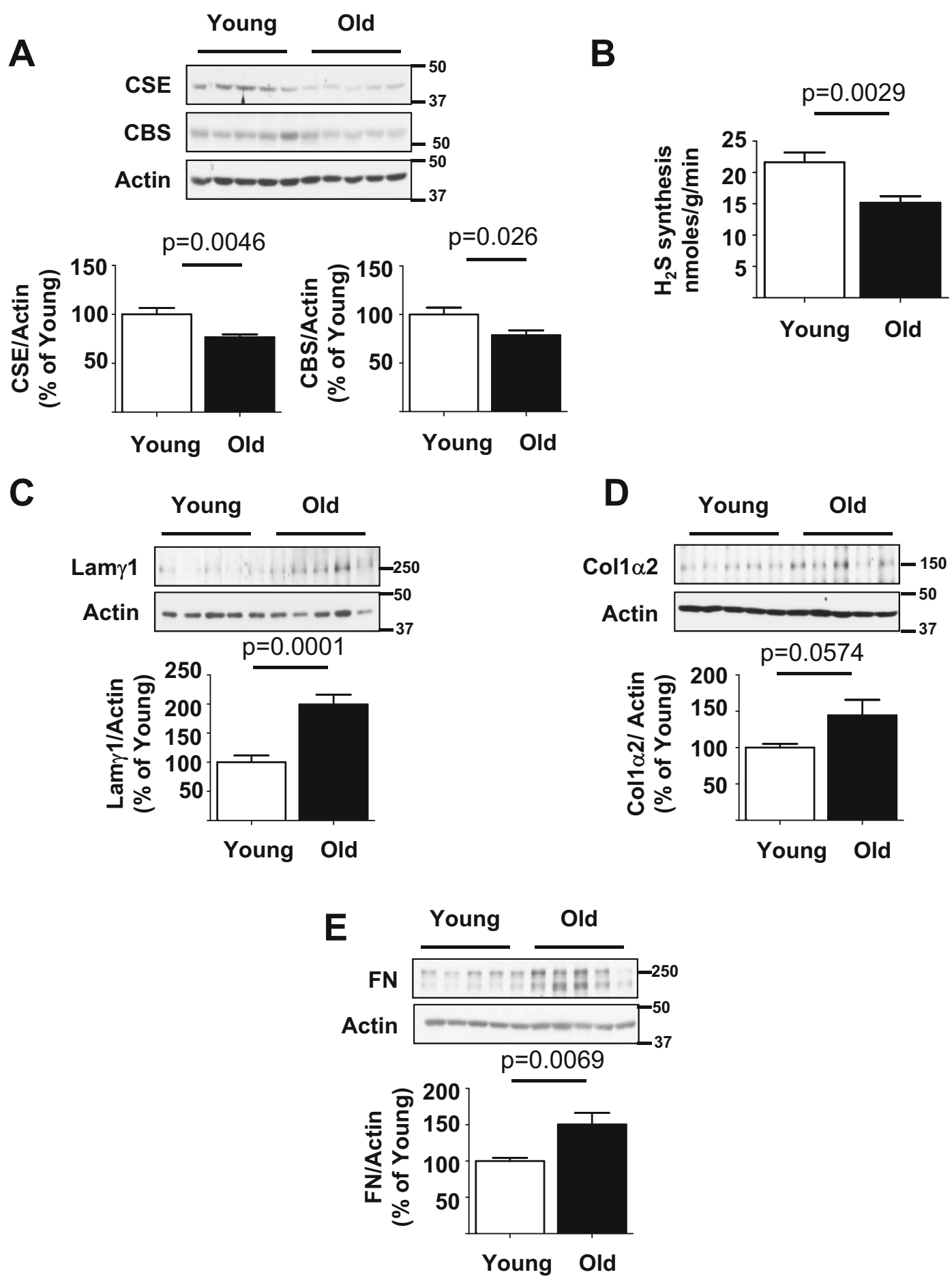

Fig. 1 Kidney cortical hydrogen sulfide $\left(\mathrm{H}_{2} \mathrm{~S}\right)$ generation is reduced in association with increase in matrix protein content old mice. a Immunoblotting of renal cortical lysates with antibodies against cystathionine $\gamma$-lyase (CSE) and cystathionine $\beta$-synthase (CBS) showed that their expression was decreased in old mice. $\mathbf{b}$ Renal cortical $\mathrm{H}_{2} \mathrm{~S}$ generation, measured as described in the
"Methods" section, was decreased in old mice. c-e Immunoblotting of renal cortical lysates with antibodies against laminin $\gamma 1$, type I collagen $\alpha 2$, and fibronectin (FN) showed increase in their expression in old mice. In $\mathbf{a}-\mathbf{e}$, data from young $(n=10)$ and old mice $(n=10)$ are shown as mean \pm SEM in histograms 

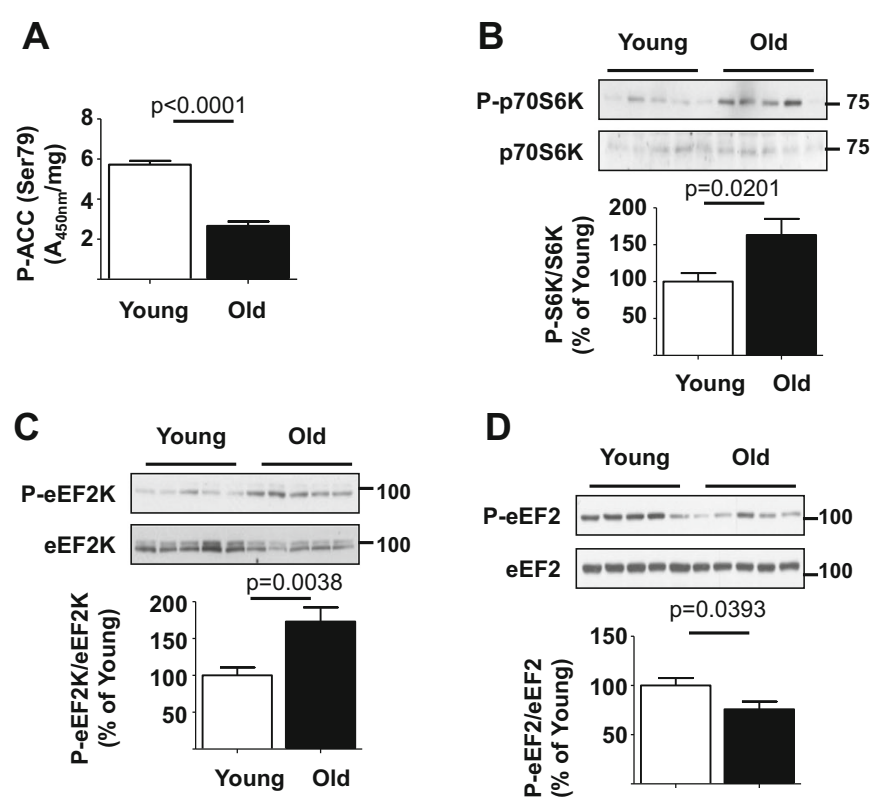

D
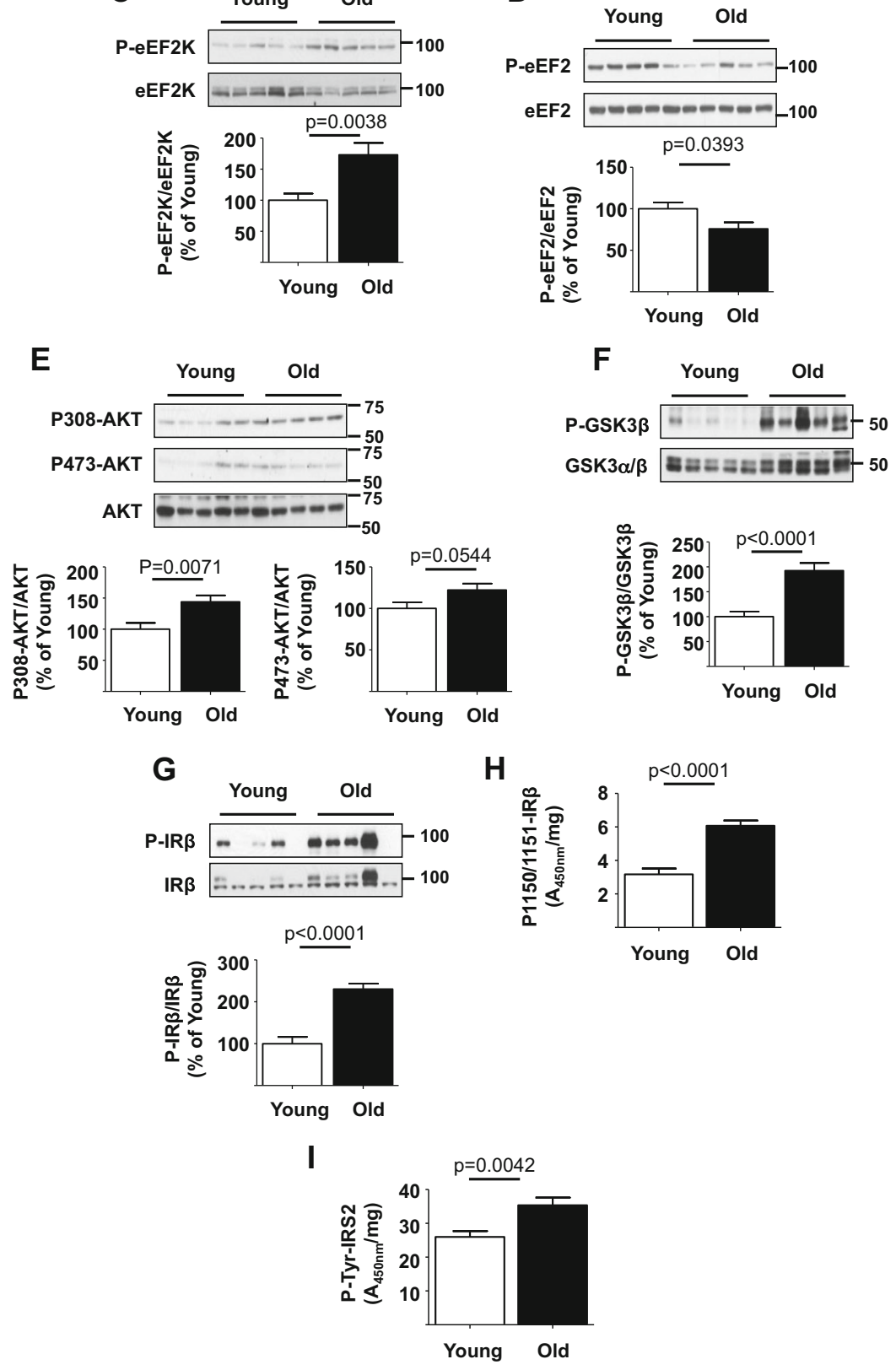
Fig. 2 Insulin receptor (IR) and signaling pathways are activated in the kidney of old mice. a Kidney cortical lysates were employed in ELISA and showed decrease in phosphorylation of acetyl Co-A carboxylase (ACC) in old vs. young mice. Immunoblotting of renal cortical lysates showed the following in old vs. young mice: $\mathbf{b}, \mathbf{c}, \mathbf{e}-\mathbf{g}$ increase in the phosphorylation of p70S6 kinase, eEF2 kinase (eEF2K), Akt, glycogen synthase kinase $3 \beta$ (GSK-3 $\beta$ ), and insulin receptor- $\beta$ (IR $\beta$ ) and $\mathbf{d}$ decrease in the phosphorylation of eukaryotic elongation factor 2 (eEF2). h, i ELISA showed increase in renal cortical tyrosine phosphorylation of IR $\beta$ and insulin receptor substrate-2 (IRS-2) in old vs. young mice. Data from young $(n=10)$ and old mice $(n=10)$ are shown as mean \pm SEM in histograms

associated glomerulosclerosis is characterized by progressive mesangial matrix expansion measured as increase in fraction mesangial volume (Sataranatarajan et al. 2012). An expanding mesangium encroaches on the glomerular capillary surface area and reduces it, thereby impairing glomerular filtration function.
Compared to control mice, the glomerular mesangium was less expanded in NaHS-treated mice; this was confirmed by morphometry which showed that NaHS administration reduced fractional mesangial volume in glomerulus by approximately $24 \%$ (Fig. 4a). There was no significant change in the overall glomerular cross sectional area (Fig. 4b). In agreement with morphological changes in mesangial matrix, kidney content of mesangial matrix protein laminin $\gamma 1$ was reduced in NaHS-treated mice relative to control mice (Fig. 4c). Kidney content of collagen I and fibronectin was also significantly decreased by NaHS treatment in old mice, suggesting reduced renal fibrosis (Fig. 4d, e). These data show that $\mathrm{H}_{2} \mathrm{~S}$ ameliorates renal fibrosis associated with aging.

$\mathrm{H}_{2} \mathrm{~S}$ stimulates AMPK and inhibits mTORC1 in kidneys of aging mice We studied signaling mechanisms underlying beneficial effect of $\mathrm{H}_{2} \mathrm{~S}$ on kidney matrix accumulation in old mice. NaHS stimulated AMPK activity by
Fig. 3 Senescence-associated secretory phenotype (SASP) occurs in the kidney of old mice. a-c Immunoblotting of kidney cortical lysates showed increase in expression of $\mathrm{p} 21$, interleukin- $1 \beta$ (IL-1 $\beta$ ), and IL- 6 in old mice in comparison with young mice. Data from young $(n=10)$ and old mice $(n=10)$ are shown as mean \pm SEM in histograms

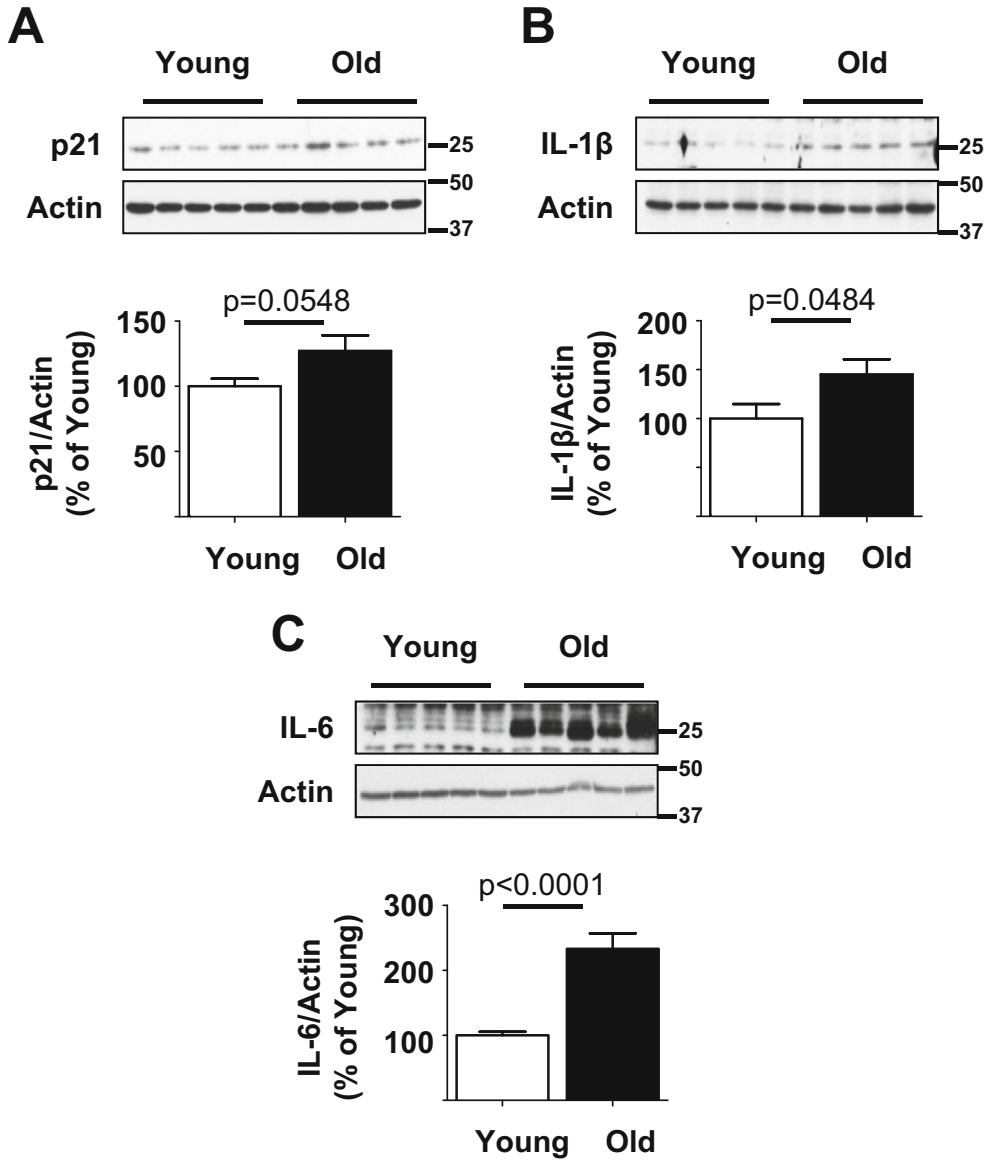


more than twofold in the kidney cortex as indicated by increase in Ser-79 phosphorylation of ACC (Fig. 5a). This was associated with reduction in Thr-389 phosphorylation of p70S6 kinase (Fig. 5b) demonstrating inhibition of mTORC1. In the NaHS-treated mice, the renal cortical Ser-366 phosphorylation of eEF2 kinase was reduced and Thr-56 phosphorylation of eEF2 was increased consistent with inhibition of elongation phase of mRNA translation (Fig. 5Dc, d). These data show that $\mathrm{H}_{2} \mathrm{~S}$ activates AMPK and inhibits key signaling events controlling protein synthesis; these reactions contribute to inhibition of aging-related increase in matrix proteins.

\section{$\mathrm{H}_{2} \mathrm{~S}$ inhibits IR-Akt axis in kidneys of aging mice NaHS} administration reduced Thr-308 phosphorylation of Akt compared to control mice, indicating inhibition of the kinase (Fig. 5e); this was confirmed by reduction in Ser9 phosphorylation of GSK $3 \beta$, an Akt substrate (Fig. 5f). Upstream of Akt, immunoblotting showed that NaHS reduced kidney cortical IR tyrosine phosphorylation relative to control mice; this was confirmed by ELISA (Fig. 5g, h). Tyrosine phosphorylation of IRS-2 in the renal cortex was also reduced in mice receiving $\mathrm{NaHS}$ in comparison with control mice (Fig. 5i). Taken together, these data show $\mathrm{H}_{2} \mathrm{~S}$ administration inhibits IR-IRS-2-Akt-mTORC1-mRNA translation (protein synthesis) signaling axis in the aging kidney.

$\mathrm{H}_{2} \mathrm{~S}$ inhibits SASP in kidneys of aging mice NaHS administration led to inhibition of mTOR activation in the aging kidney (Fig. 5b). Since mTOR inhibition can ameliorate SASP (Wang et al. 2017), we examined NaHS effect on SASP. As shown in Fig. 6a-c, NaHS reduced the levels of p 21 , IL-1 $\beta$, and IL- 6 in the kidney cortex relative to control mice, suggesting inhibition of SASP.

$\mathrm{H}_{2} \mathrm{~S}$ reduces urinary albumin excretion and preserves renal clearance function in aging mice Preventing losses of plasma albumin during glomerular filtration is an important function of the kidney. Thus, a rise in urinary albumin to creatinine ratio (ACR) is an index of kidney injury. Urinary ACR rose significantly during the study period in control mice relative to the baseline value at the time of randomization. In contrast to the control group, in the NaHS group urinary ACR did not change significantly from the baseline; it was significantly lower than in control mice at every month
(Fig. 7a). Serum cystatin C level varies inversely with glomerular filtration rate and is employed as an index of renal waste clearance function in aging studies in mice (Kume et al. 2010) (Sataranatarajan et al. 2012) (Song et al. 2009). At the end of the study, relative to mice in the control group, serum cystatin $\mathrm{C}$ values were significantly lower in mice receiving NaHS (Fig. 7b). Collectively, these data show that $\mathrm{H}_{2} \mathrm{~S}$ reduces albuminuria in aging mice. NaHS-induced reduction in serum cystatin $\mathrm{C}$ implies an improvement in filtration function or waste clearance function of the kidney.

$\mathrm{NaHS}$ administration does not affect kidney $\mathrm{H}_{2} \mathrm{~S}$ metabolism in aging mice Administration of NaHS increased plasma free sulfide levels (Suppl. Fig. S4A). However, it did not affect kidney cortical expression of CBS and CSE proteins or generation of $\mathrm{H}_{2} \mathrm{~S}$ (Suppl. Fig. S4B, C). Thus, there was no feedback inhibition of expression of $\mathrm{H}_{2} \mathrm{~S}$-synthesizing enzymes CBS and CSE or its generation in the kidney by the exogenously administered NaHS.

\section{Discussion}

Our data show that $\mathrm{H}_{2} \mathrm{~S}$ deficiency is an important mechanism contributing to aging-related changes in kidney structure and function. In the kidney of old mice, $\mathrm{H}_{2} \mathrm{~S}$ deficiency occurs due to reduced expression of enzymes that generate the gas, i.e., CSE and CBS. Administration of NaHS to aging mice ameliorates both structural and functional aspects of renal aging. The mechanism underlying beneficial effects of $\mathrm{H}_{2} \mathrm{~S}$ on renal aging involves inhibition of fundamental aging processes such as activation of IR and signaling pathways involving mTOR that stimulate protein synthesis.

Since increased protein synthesis contributes to matrix protein accumulation, we focused on signaling pathways that regulate mRNA translation, a rate-limiting step in protein synthesis (Holz et al. 2005). mTORC1 governs initiation and elongation phases of mRNA translation (Kasinath et al. 2009). Activation of Akt leads to stimulation of mTORC1 in at least two ways. First, it phosphorylates Ser-487 on the serine threonine-rich loop of the $\alpha 1$ subunit of AMPK and inhibits its activity (Hawley et al. 2014). Decreased AMPK activity is associated with mTORC1 activation in the kidney in diabetes (Eid et al. 2010; Lee et al. 2007; Sharma et al. 2008). The second mechanism involves proline-rich Akt substrate 40 


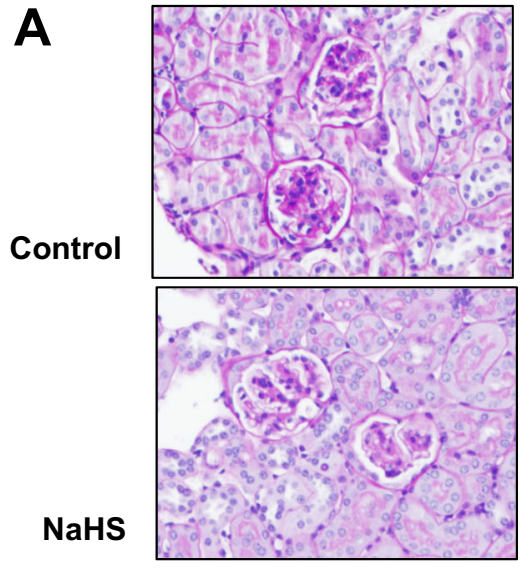

C
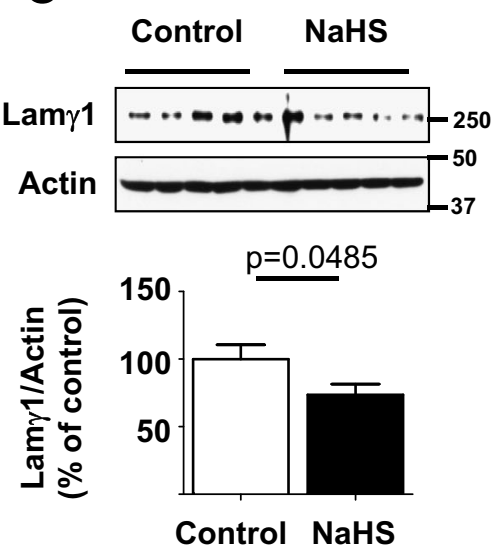

B

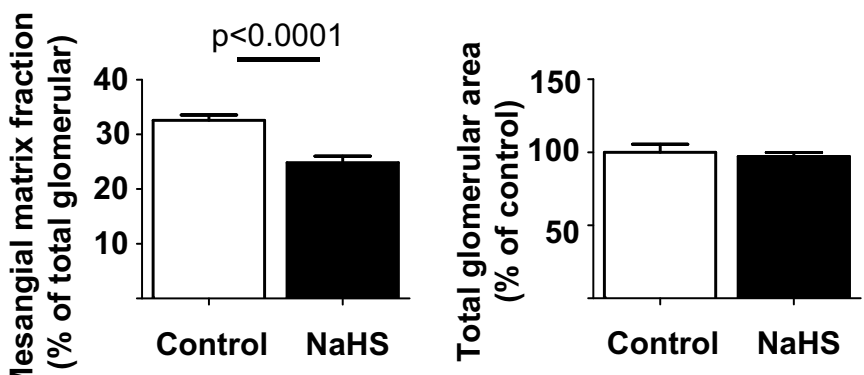

D
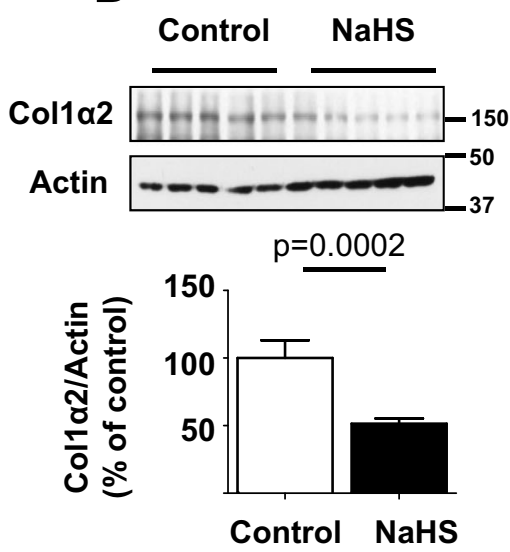

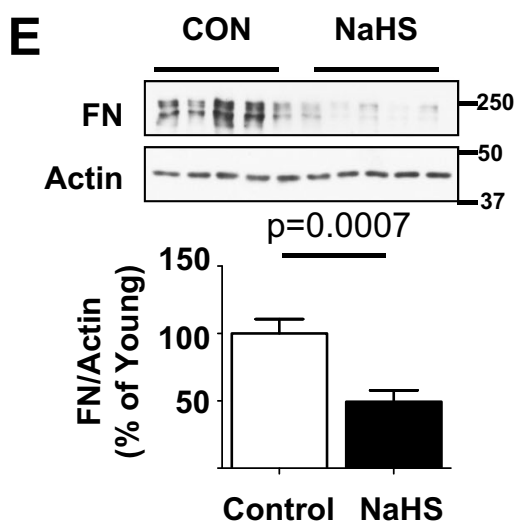

Fig. 4 Hydrogen sulfide ameliorates extracellular matrix accumulation in old mice. 18-19-month-old mice were randomized to receive NaHS in drinking water (NaHS, $n=20$ mice) or water alone (control, $n=14$ mice) and followed for 5 months. a NaHS reduced glomerulosclerosis and glomerular mesangial matrix fraction expressed as percent of glomerular area. b Total glomerular

(PRAS40). PRAS40 exists in a complex with and constitutively inhibits mTORC1; following phosphorylation area was unchanged between the two groups. c-e Immunoblotting of renal cortical lysates showed reduction in expression of laminin $\gamma 1$, type I collagen $\alpha 2$, and fibronectin in mice that received $\mathrm{NaHS}$ vs. control mice. Data are shown as mean \pm SEM in histograms

by Akt, PRAS40 dissociates from mTORC1 allowing it to become active (Sancak et al. 2007). Renal mTORC1 is 
Fig. 5 Hydrogen sulfide inhibits activation of insulin receptor (IR) and signaling pathways in the kidney of old mice. 18-19-month old mice were randomized to receive NaHS in drinking water (NaHS, $n=20$ mice) or water alone (control, $n=14$ mice) and followed for 5 months. Compared to control mice, the kidney cortical lysates from mice that received NaHS showed the following: a increase in phosphorylation of ACC by ELISA; b, c, e- $\mathbf{g}$ decrease in phosphorylation of p70S6 kinase, eEF2 kinase (eEF2K), Akt, GSK-3 $\beta$, and IR $\beta$; $\mathbf{d}$ increase in phosphorylation of eEF2 by immunoblotting; and $\mathbf{h}, \mathbf{i}$ decrease in renal cortical tyrosine phosphorylation of IR $\beta$ and IRS2 by ELISA. Data are shown as mean \pm SEM in histograms
A
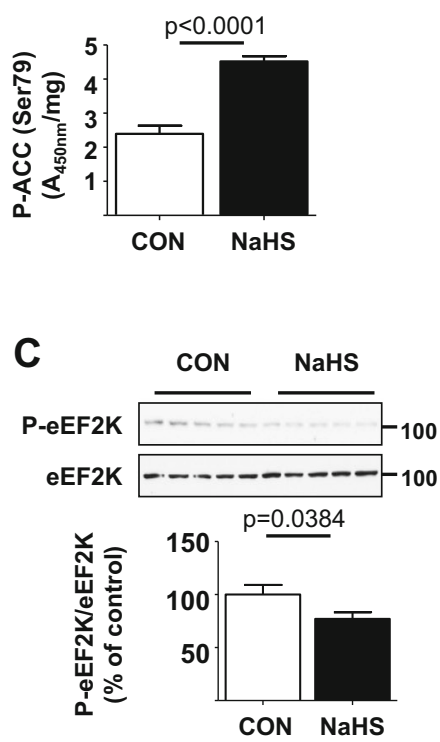

E
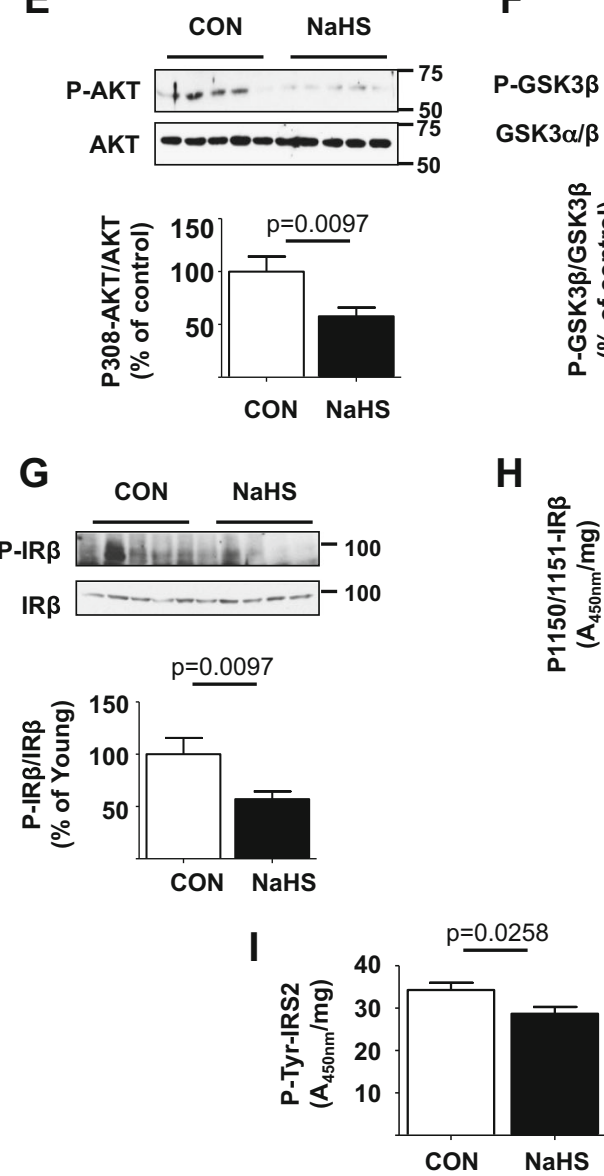
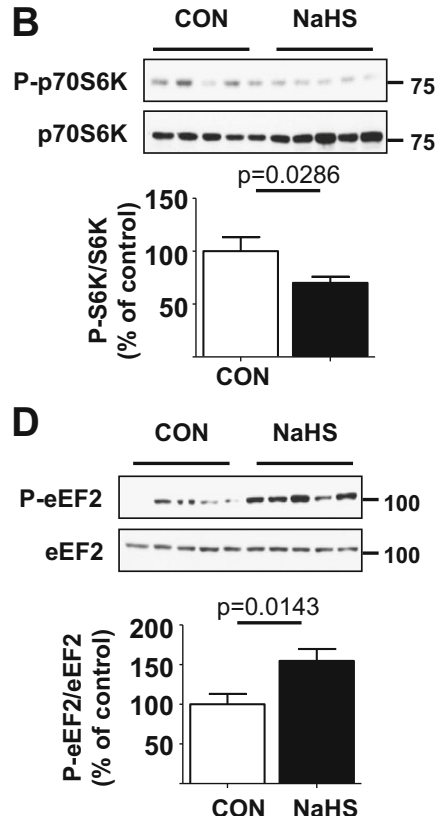

$\mathbf{F}$
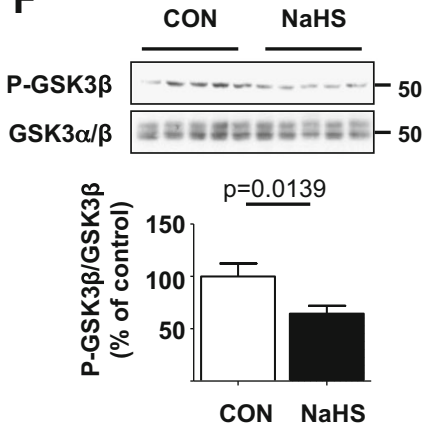

H

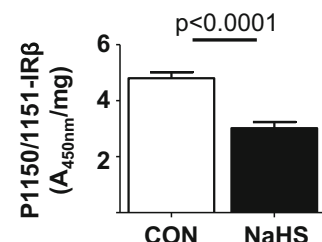


Fig. 6 Hydrogen sulfide ameliorates SASP in the kidney of old mice. 18-19-month-old mice were randomized to receive $\mathrm{NaHS}$ in drinking water (NaHS, $n=20$ mice) or water alone (control, $n=14$ mice) and followed for 5 months. a-c Immunoblotting of renal cortical lysates showed decrease in the expression of $\mathrm{p} 21$, IL- $1 \beta$, and IL6 in mice that received NaHS vs. control mice. Data are shown as mean \pm SEM in histograms

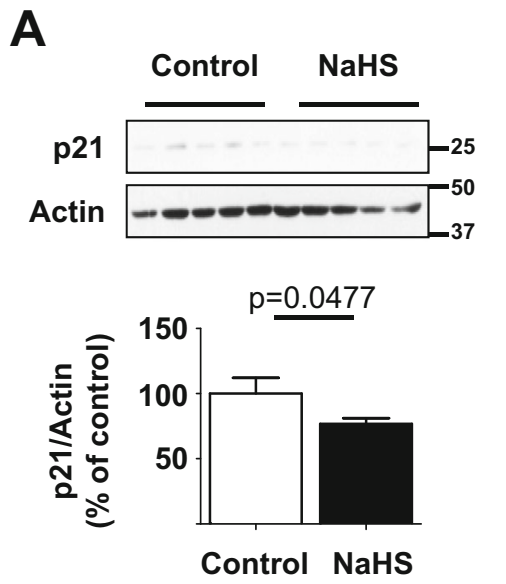

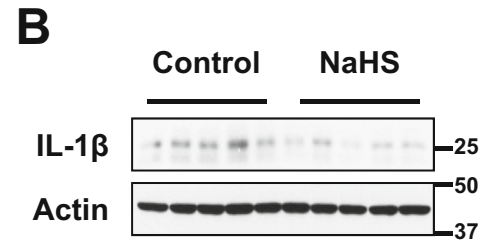

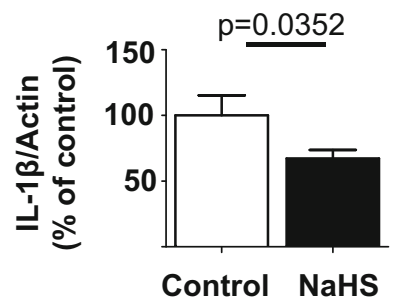

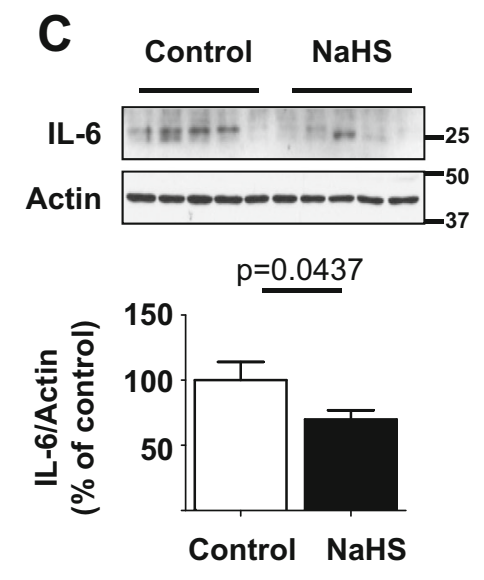

activated in early diabetes and its inhibition with rapamycin ameliorates associated kidney injury (Eid et al. 2010; Lee et al. 2007; Sataranatarajan et al. 2007; Sharma et al. 2008). $\mathrm{H}_{2} \mathrm{~S}$ and phosphodiesterase-5 inhibitor tadalafil, which promotes $\mathrm{H}_{2} \mathrm{~S}$ release, ameliorate high glucose-induced matrix increment in renal epithelial cells by stimulating AMPK and inhibiting mTORC1 (Lee et al. 2015; Lee et al. 2012). Our data show that aging is also associated with inhibition of AMPK and stimulation of $\mathrm{mTORC} 1$, which leads to stimulation of mRNA translation; these events facilitate increase in the synthesis of matrix proteins. Administration of $\mathrm{H}_{2} \mathrm{~S}$ stimulated AMPK to inhibit mTORC1 and translation, contributing to reduced synthesis of matrix proteins and renal fibrosis. Our search for upstream regulators of Akt led to IR activation. Increase in tyrosine phosphorylation of IRS-2, a protein that docks with cytoplasmic domain of IR and amplifies its signaling, further supported IR activation in the aging kidney. Our previous studies have shown that IR activates Akt-mTORC1 axis in renal

epithelial cells leading to increase in the synthesis of proteins including matrix proteins (Bhandari et al. 2001; Mariappan et al. 2007). IR-related signaling pathways have been implicated in mediating aging-related changes (Bartke et al. 2013; Bluher et al. 2003; Taguchi et al. 2007). Our study showed that aging-associated $\mathrm{H}_{2} \mathrm{~S}$ deficiency is upstream of IR activation in the kidney because exogenous administration inhibited it. $\mathrm{H}_{2} \mathrm{~S}$ appears to regulate fundamental aging processes that promote IR activation. Future studies will need to address the mechanism linking $\mathrm{H}_{2} \mathrm{~S}$ deficiency to IR activation in the aging kidney.

During aging, subpopulations of cells in tissues undergo permanent cell cycle arrest termed cellular senescence (Childs et al. 2015). One mechanism of senescence could involve the following scenario. In proliferating cells, aging-associated telomere erosion elicits a DNA damage response in which ataxia telangiectasia mutated, a serine threonine kinase, is brought to the eroded telomeres; this is followed by stabilization of p53 and 
A
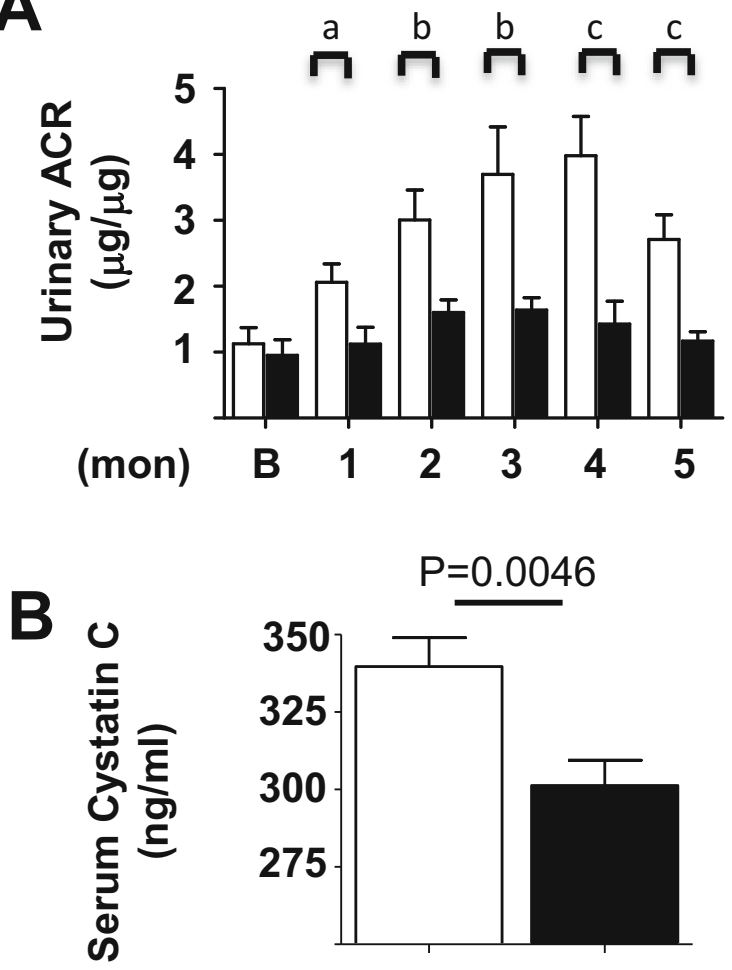

Control NaHS

Fig. 7 Hydrogen sulfide inhibits age-related increase in albuminuria and it decreases serum cystatin $\mathrm{C}$ in old mice. Mice, 1819 months of age, were randomized to receive NaHS in drinking water or water alone (control) and followed for 5 months. (A) Urinary albumin to creatinine ratio (ACR) increased in control mice during the study period which was prevented by NaHS (a) $p<0.05$, (b) $p<0.01$, and (c) $p<0.001$. (B) Serum cystatin C was lower in NaHS-treated mice vs. control mice at the end of the study. Data from control $(n=13-20)$ and NaHS-treated mice $(n=$ 18-20) are shown as mean \pm SEM in histograms

increase in the expression of its target p21 (Childs et al. 2015). The downstream events involve p21 inhibiting cyclin-dependent kinase 2-mediated $\mathrm{Rb}$ inactivation, which maintains cells in an arrest mode (ibid). Senescent cells assume a phenotype of secreting pro-inflammatory cytokines, e.g., IL-1 and IL-6 (SASP), with autocrine or paracrine effects promoting inflammation, epithelial to mesenchymal transition, and even transforming target cells into a secretory phenotype (SASP on SASP) (Childs et al. 2015; Nelson et al. 2012). Activation of mTORC1 has been linked to SASP, and rapamycin inhibits the process (Iglesias-Bartolome et al. 2012; Wang et al. 2017). Removal of cells manifesting SASP from the kidneys ameliorates aging-associated renal changes (Baar et al. 2017; Baker et al. 2016). Renal cortical
mTOR activation in aging kidneys was associated with increase in the expression of $\mathrm{p} 21$, IL-1 $\beta$, and IL-6, features of SASP. These changes were abolished by exogenous administration of $\mathrm{H}_{2} \mathrm{~S}$. Our data make the novel observation that $\mathrm{H}_{2} \mathrm{~S}$ deficiency contributes to SASP by stimulation of mTOR in the aging kidney.

Our study identifies a novel mechanism in renal aging by identifying $\mathrm{H}_{2} \mathrm{~S}$ deficiency as a contributory factor and linking it to signaling events involving inhibition of AMPK and activation of IR-Akt-mTORC1 axis. We administered NaHS by the oral route for 5 months starting at the age of 18-19 months that reversed signaling changes and resulted in these salutary outcomes. Another group of investigators recently reported that intraperitoneal administration of NaHS to 16-month-old mice for 10 weeks stimulates well-known anti-oxidant pathways involving Nrf2 to ameliorate aging-related changes in the kidney (Hou et al. 2016); however, that study did not explore the status of $\mathrm{H}_{2} \mathrm{~S}$ or signaling mechanisms including IR activation. Our exploration of signaling identified inhibition of AMPK as a key feature of renal aging; administration of $\mathrm{H}_{2} \mathrm{~S}$ stimulates AMPK activity that leads to mTORC1 inhibition and reduction in matrix protein synthesis. These detailed observations in signaling pathways governing protein synthesis identify potential novel sites for intervention to ameliorate aging-related changes in the kidney.

Our study has limitations. We did not study other properties of $\mathrm{H}_{2} \mathrm{~S}$ that may be potentially involved in amelioration of kidney injury, e.g., mitochondrial biogenesis and regulation of epigenetics (Weber et al. 2017). In future, we will need to address the role of $\mathrm{H}_{2}$ $\mathrm{S}$ in recently reported mechanisms of kidney injury in aging, e.g., podocyte deficiency leading to glomerulosclerosis, mitochondrial dysfunction, and fructose metabolism (Hodgin et al. 2015; RoncalJimenez et al. 2016; Sweetwyne et al. 2017). Additionally, we did not test whether $\mathrm{H}_{2} \mathrm{~S}$ prolongs life span in mice as has been shown in C elegans (Miller et al. 2011; Miller and Roth 2007). The life span-prolonging effect of dietary restriction depends on increase in generation of H2S in worms and yeast (Hine et al. 2015).

Our study adds aging-related kidney dysfunction to the list of conditions ameliorated by $\mathrm{H}_{2} \mathrm{~S}$, i.e., acute kidney injury (Bos et al. 2013; Dugbartey et al. 2016), diabetes (van den Born et al. 2016; Yuan et al. 2011; Zhou et al. 2014), obstructive uropathy (Song et al. 2014), hypertension (Weber et al. 2017), and hyperhomocysteinemia (Sen et al. 2012). The odor of $\mathrm{H}_{2} \mathrm{~S}$ is 
clearly unacceptable for humans although we employed $\mathrm{NaHS}$ as a proof of concept. Other formulations that release $\mathrm{H}_{2} \mathrm{~S}$ are available for future testing (Wallace and Wang 2015). Phosphodiesterase-5 inhibitor, tadalafil, stimulates $\mathrm{H}_{2} \mathrm{~S}$ generation in kidney epithelial cells to inhibit high glucose-induced increase in matrix proteins (Lee et al. 2015); it is routinely employed in the treatment of erectile dysfunction and could be considered a candidate for studies in ameliorating aging-associated kidney impairment in humans.

Author contributions BSK, VG, JN, AS, and RS designed the study and analyzed the data. HJL, DF, SO, JB, and CK performed the experiments. All authors participated in data analysis and interpretation. BSK wrote the manuscript; all authors approved it.Funding informationA Pilot Study Grant to BSK from the Nathan Shock Center of Excellence in Basic Biology of Aging (NIH 1P30-AG013319) supported this work. This work was also supported by grants from the Veterans Affairs Biomedical Laboratory Research and Development Service (I01BX001340 to BSK, 5I01BX000926 to GGC, I01BX002211 to VG, II01BX001641 to RS) and the NIH (DK50190 to GGC, HL113303 to CGK, AG050797 to ABS). DF is supported by the American Heart Association (15GRNT25700363). GGC is a recipient of a Veterans Affairs Senior Research Career Scientist Award (IK6BX003611).

\section{Compliance with ethical standards}

The Institutional Animal Care and Utilization Committee of the University of Texas Health at San Antonio approved the animal studies.

Conflict of interest CGK has intellectual property for hydrogen sulfide measurement and biomarker and also has equity interest in Innolyzer LLC and Theravasc Inc. J.L.B. is a consultant and cofounder of Probetex, Inc. with ownership interests. All other authors declare that they have no conflict of interest.

\section{References}

Abe K, Kimura H (1996) The possible role of hydrogen sulfide as an endogenous neuromodulator. J Neurosci 16(3):10661071

Acosta JC, Banito A, Wuestefeld T, Georgilis A, Janich P, Morton JP et al (2013) A complex secretory program orchestrated by the inflammasome controls paracrine senescence. Nat Cell Biol 15(8):978-990

Baar MP, Brandt RM, Putavet DA, Klein JD, Derks KW, Bourgeois BR et al (2017) Targeted apoptosis of senescent cells restores tissue homeostasis in response to chemotoxicity and aging. Cell 169(1):132-147 e116

Baker DJ, Childs BG, Durik M, Wijers ME, Sieben CJ, Zhong J et al (2016) Naturally occurring p16(Ink4a)-positive cells shorten healthy lifespan. Nature 530(7589):184-189
Barsha G, Denton KM, Mirabito Colafella KM (2016) Sex- and age-related differences in arterial pressure and albuminuria in mice. Biol Sex Differ 7:57

Bartke A, Sun LY, Longo V (2013) Somatotropic signaling: tradeoffs between growth, reproductive development, and longevity. Physiol Rev 93(2):571-598

Bhandari BK, Feliers D, Duraisamy S, Stewart JL, Gingras AC, Abboud HE et al (2001) Insulin regulation of protein translation repressor 4E-BP1, an eIF4E-binding protein, in renal epithelial cells. Kidney Int 59(3):866-875

Bluher M, Kahn BB, Kahn CR (2003) Extended longevity in mice lacking the insulin receptor in adipose tissue. Science 299(5606):572-574

Bos EM, Wang R, Snijder PM, Boersema M, Damman J, Fu M et al (2013) Cystathionine gamma-lyase protects against renal ischemia/reperfusion by modulating oxidative stress. $\mathrm{J}$ Am Soc Nephrol 24(5):759-770

Childs BG, Durik M, Baker DJ, van Deursen JM (2015) Cellular senescence in aging and age-related disease: from mechanisms to therapy. Nat Med 21(12):1424-1435

Dugbartey GJ, Peppone LJ, de Graaf IA (2016) An integrative view of cisplatin-induced renal and cardiac toxicities: molecular mechanisms, current treatment challenges and potential protective measures. Toxicology 371:58-66

Eid AA, Ford BM, Block K, Kasinath BS, Gorin Y, GhoshChoudhury G et al (2010) AMP-activated protein kinase (AMPK) negatively regulates Nox4-dependent activation of p53 and epithelial cell apoptosis in diabetes. J Biol Chem 285(48):37503-37512

Feliers D, Lee HJ, Kasinath BS (2016) Hydrogen sulfide in renal physiology and disease. Antioxid Redox Signal 25(13):720 731

Ha TS, Barnes JL, Stewart JL, Ko CW, Miner JH, Abrahamson DR et al (1999) Regulation of renal laminin in mice with type II diabetes. J Am Soc Nephrol 10(9):1931-1939

Hawley SA, Ross FA, Gowans GJ, Tibarewal P, Leslie NR, Hardie DG (2014) Phosphorylation by Akt within the ST loop of AMPK-alpha1 down-regulates its activation in tumour cells. Biochem J 459(2):275-287

Hine C, Harputlugil E, Zhang Y, Ruckenstuhl C, Lee BC, Brace L et al (2015) Endogenous hydrogen sulfide production is essential for dietary restriction benefits. Cell 160(1-2):132144

Hodgin JB, Bitzer M, Wickman L, Afshinnia F, Wang SQ, O'Connor C et al (2015) Glomerular aging and focal global glomerulosclerosis: a podometric perspective. J Am Soc Nephrol 26(12):3162-3178

Holz MK, Ballif BA, Gygi SP, Blenis J (2005) mTOR and S6K1 mediate assembly of the translation preinitiation complex through dynamic protein interchange and ordered phosphorylation events. Cell 123(4):569-580

Hou CL, Wang MJ, Sun C, Huang Y, Jin S, Mu XP et al (2016) Protective effects of hydrogen sulfide in the ageing kidney. Oxidative Med Cell Longev 2016:7570489

Iglesias-Bartolome R, Patel V, Cotrim A, Leelahavanichkul K, Molinolo AA, Mitchell JB, Gutkind JS (2012) mTOR inhibition prevents epithelial stem cell senescence and protects from radiation-induced mucositis. Cell Stem Cell 11(3):401414

Kasinath BS, Feliers D, Sataranatarajan K, Ghosh Choudhury G, Lee MJ, Mariappan MM (2009) Regulation of mRNA 
translation in renal physiology and disease. Am J Physiol Renal Physiol 297(5):F1153-F1165

Kume S, Uzu T, Horiike K, Chin-Kanasaki M, Isshiki K, Araki S et al (2010) Calorie restriction enhances cell adaptation to hypoxia through Sirt1-dependent mitochondrial autophagy in mouse aged kidney. J Clin Invest 120(4):1043-1055

Lee MJ, Feliers D, Mariappan MM, Sataranatarajan K, Mahimainathan L, Musi N et al (2007) A role for AMPactivated protein kinase in diabetes-induced renal hypertrophy. Am J Physiol Renal Physiol 292(2):F617-F627

Lee HJ, Mariappan MM, Feliers D, Cavaglieri RC, Sataranatarajan K, Abboud HE et al (2012) Hydrogen sulfide inhibits high glucose-induced matrix protein synthesis by activating AMP-activated protein kinase in renal epithelial cells. J Biol Chem 287(7):4451-4461

Lee HJ, Feliers D, Mariappan MM, Sataranatarajan K, Choudhury GG, Gorin Y, Kasinath BS (2015) Tadalafil integrates nitric oxide-hydrogen sulfide signaling to inhibit high glucoseinduced matrix protein synthesis in podocytes. J Biol Chem 290(19):12014-12026

Mariappan MM, Feliers D, Mummidi S, Choudhury GG, Kasinath BS (2007) High glucose, high insulin, and their combination rapidly induce laminin-betal synthesis by regulation of mRNA translation in renal epithelial cells. Diabetes 56(2): 476-485

Miller DL, Roth MB (2007) Hydrogen sulfide increases thermotolerance and lifespan in Caenorhabditis elegans. Proc Natl Acad Sci U S A 104(51):20618-20622

Miller DL, Budde MW, Roth MB (2011) HIF-1 and SKN-1 coordinate the transcriptional response to hydrogen sulfide in Caenorhabditis elegans. PLoS One 6(9):e25476

Nelson G, Wordsworth J, Wang C, Jurk D, Lawless C, MartinRuiz C, von Zglinicki T (2012) A senescent cell bystander effect: senescence-induced senescence. Aging Cell 11(2): 345-349

Paul BD, Snyder SH (2012) H(2)S signalling through protein sulfhydration and beyond. Nat Rev Mol Cell Biol 13(8): 499-507

Redpath NT, Foulstone EJ, Proud CG (1996) Regulation of translation elongation factor-2 by insulin via a rapamycinsensitive signalling pathway. EMBO J 15(9):2291-2297

Roncal-Jimenez CA, Ishimoto T, Lanaspa MA, Milagres T, Hernando AA, Jensen T et al (2016) Aging-associated renal disease in mice is fructokinase dependent. Am J Physiol Renal Physiol 311(4):F722-F730

Sancak Y, Thoreen CC, Peterson TR, Lindquist RA, Kang SA, Spooner E et al (2007) PRAS40 is an insulin-regulated inhibitor of the mTORC1 protein kinase. Mol Cell 25(6): 903-915

Sataranatarajan K, Mariappan MM, Lee MJ, Feliers D, Choudhury GG, Barnes JL, Kasinath BS (2007) Regulation of elongation phase of mRNA translation in diabetic nephropathy: amelioration by rapamycin. Am J Pathol 171(6):1733-1742

Sataranatarajan K, Feliers D, Mariappan MM, Lee HJ, Lee MJ, Day RT et al (2012) Molecular events in matrix protein metabolism in the aging kidney. Aging Cell 11(6):1065-1073

Sataranatarajan K, Ikeno Y, Bokov A, Feliers D, Yalamanchili H, Lee HJ et al (2016) Rapamycin increases mortality in $\mathrm{db} / \mathrm{db}$ mice, a mouse model of type 2 diabetes. J Gerontol A Biol Sci Med Sci 71(7):850-857
Sen U, Pushpakumar S (2016) Mini-review: diabetic renal complications, a potential stinky remedy. Am J Physiol Renal Physiol 310(2):F119-F122

Sen U, Basu P, Abe OA, Givvimani S, Tyagi N, Metreveli N, Shah KS, Passmore JC, Tyagi SC (2009) Hydrogen sulfide ameliorates hyperhomocysteinemia-associated chronic renal failure. Am J Physiol Renal Physiol 297(2):F410-F419

Sen U, Sathnur PB, Kundu S, Givvimani S, Coley DM, Mishra PK et al (2012) Increased endogenous H2S generation by CBS, CSE, and 3MST gene therapy improves ex vivo renovascular relaxation in hyperhomocysteinemia. Am J Physiol Cell Physiol 303(1):C41-C51

Sharma K, Ramachandrarao S, Qiu G, Usui HK, Zhu Y, Dunn SR et al (2008) Adiponectin regulates albuminuria and podocyte function in mice. J Clin Invest 118(5):1645-1656

Shen X, Pattillo CB, Pardue S, Bir SC, Wang R, Kevil CG (2011) Measurement of plasma hydrogen sulfide in vivo and in vitro. Free Radic Biol Med 50(9):1021-1031

Song S, Meyer M, Turk TR, Wilde B, Feldkamp T, Assert R et al (2009) Serum cystatin C in mouse models: a reliable and precise marker for renal function and superior to serum creatinine. Nephrol Dial Transplant 24(4):1157-1161

Song K, Wang F, Li Q, Shi YB, Zheng HF, Peng H et al (2014) Hydrogen sulfide inhibits the renal fibrosis of obstructive nephropathy. Kidney Int 85(6):1318-1329

Sweetwyne MT, Pippin JW, Eng DG, Hudkins KL, Chiao YA, Campbell MD et al (2017) The mitochondrial-targeted peptide, SS-31, improves glomerular architecture in mice of advanced age. Kidney Int 91(5):1126-1145

Taguchi A, Wartschow LM, White MF (2007) Brain IRS2 signaling coordinates life span and nutrient homeostasis. Science 317(5836):369-372

van den Born JC, Hammes HP, Greffrath W, van Goor H, Hillebrands JL et al (2016) Gasotransmitters in vascular complications of diabetes. Diabetes 65(2):331-345

Wallace JL, Wang R (2015) Hydrogen sulfide-based therapeutics: exploiting a unique but ubiquitous gasotransmitter. Nat Rev Drug Discov 14(5):329-345

Wang R (2012) Shared signaling pathways among gasotransmitters. Proc Natl Acad Sci U S A 109(23):88018802

Wang R, Yu Z, Sunchu B, Shoaf J, Dang I, Zhao S et al (2017) Rapamycin inhibits the secretory phenotype of senescent cells by a Nrf2-independent mechanism. Aging Cell 16(3): 564-574

Weber GJ, Pushpakumar SB, Sen U (2017) Hydrogen sulfide alleviates hypertensive kidney dysfunction through an epigenetic mechanism. Am J Physiol Heart Circ Physiol 312(5): H874-H885

Yang G, Wu L, Jiang B, Yang W, Qi J, Cao K et al (2008) H2S as a physiologic vasorelaxant: hypertension in mice with deletion of cystathionine gamma-lyase. Science 322(5901):587-590

Yuan P, Xue H, Zhou L, Qu L, Li C, Wang Z et al (2011) Rescue of mesangial cells from high glucose-induced over-proliferation and extracellular matrix secretion by hydrogen sulfide. Nephrol Dial Transplant 26(7):2119-2126

Zhou X, Feng Y, Zhan Z, Chen J (2014) Hydrogen sulfide alleviates diabetic nephropathy in a streptozotocin-induced diabetic rat model. J Biol Chem 289(42):28827-28834 